[Article]

\title{
腺嘌呤与富马酸共晶体的太赫兹光谱分析
}

\author{
方虹霞 张 琪 张慧丽 杜 勇 ${ }^{*}$ 洪 治 \\ (中国计量学院太赫兹技术与应用研究所, 杭州 310018)
}

\begin{abstract}
摘要: 利用太赫兹时域光谱(THz-TDS)技术在室温下对腺嘌呤、富马酸及两者的共晶体进行测量, 实验结果显 示腺嘌呤与富马酸共晶体在 0.92、1.24、1.52 THz 处有明显的吸收峰, 与腺嘌呤和富马酸不同, 表明共晶体物相 结构不同于原料. 根据腺嘌呤分子氢键供体与受体的结构特点, 使用密度泛函理论(DFT)对腺嘌呤与富马酸三 种可能的共晶体结构进行模拟. 结果显示其中一种可能的共晶体结构在 0.89、1.16、1.41 THz 处存在特征吸收 峰, 与实验结果较好吻合. 由此判断腺嘌呤与富马酸共晶体氢键形成位置为腺嘌呤的氨基与富马酸其中一个羧 酸的碳氧双键形成氢键, 而此羧酸的羟基与腺嘌呤六元环上的邻位氮原子形成第二处氢键. 本文还结合理论模 拟的结果对腺嘌呤与富马酸共晶体的特征吸收峰对应的相关振动模式进行了归属.
\end{abstract}

关键词: 腺嘌呤; 富马酸; 共晶体; 氢键; 太赫兹时域光谱; 密度泛函理论 中图分类号: 0641; 0657.3

\section{Terahertz Spectroscopic Analysis of Adenine and Fumaric Acid Cocrystals}

\author{
FANG Hong-Xia ZHANG Qi ZHANG Hui-Li DU Yong* HONG Zhi
}

(Centre for Terahertz Research, China Jiliang University, Hangzhou 310018)

\begin{abstract}
The absorption spectra of adenine, fumaric acid, and their cocrystal were measured using terahertz time-domain spectroscopy (THz-TDS) at room temperature. Experimental results show that they all have distinct fingerprint spectra in the terahertz region. The absorption peaks observed in the terahertz spectra of the cocrystal were at $0.92,1.24$, and $1.52 \mathrm{THz}$. These are very different from the corresponding reagents. Based on the characteristic hydrogen donor and/or acceptor behavior of adenine, density functional theory (DFT) was used to simulate three possible theoretical cocrystal structures with a focus on hydrogen bond formation between adenine and fumaric acid. The theoretical result shows that one of three possible simulated cocrystal structures had absorption peaks at $0.89,1.16$, and $1.41 \mathrm{THz}$, which is in agreement with the terahertz experimental result. Therefore, the structure of the cocrystal was confirmed wherein the first hydrogen bond is formed between the amino group of adenine and the hydroxyl group of fumaric acid. The second hydrogen bond is formed between the nitrogen atom of the nitrogen ring in adenine and the carbonyl group of fumaric acid. The characteristic absorption bands of the cocrystal between adenine and fumaric acid are also assigned based on the simulation results from the DFT calculation.
\end{abstract}

Key Words: Adenine; Fumaric acid; Cocrystal; Hydrogen bond; Terahertz time-domain spectroscopy; Density functional theory

Received: November 5, 2014; Revised: December 22, 2014; Published on Web: December 23, 2014.

*Corresponding author. Email: yongdu@cjlu.edu.cn; Tel: +86-571-86875674.

The project was supported by the National Natural Science Foundation of China (21205110).

国家自然科学基金(21205110)资助项目

(c) Editorial office of Acta Physico-Chimica Sinica 


\section{1 引言}

DNA 与许多化合物或天然小分子相互作用形 成氢键, 一直是化学治疗的热门研究方向, ${ }^{1}$ 小分子 通过插入沟槽或静电模式的方式与 DNA 结合已促 进了多种新型抗癌 ${ }^{2}$ 及抗菌 ${ }^{3}$ 药物的发展. 腺嘌呤作 为 DNA 的一种碱基, 它与其他小分子的相互作用也 已被研究报道. Thompson 等 ${ }^{4}$ 使用 X射线衍射技术 检测了腺嘌呤与富马酸、马来酸及琥珀酸的共晶 体; Byres 等 ${ }^{5}$ 使用 $X$ 射线衍射技术发现腺嘌呤和质 子化腺嘌呤能与 2,6-二差茎甲酸、3,5-二羟苯甲酸、 已二酸等形成共晶体; Perumaila 等 ${ }^{6}$ 也检测了腺嘌 呤与苯甲酸共晶体; 此外, 腺嘌呤还能与水杨酸、7,8 草酸、 ${ }^{9}$ 等形成共晶体. 观察以上共晶体可发现参与 共晶形成的腺嘌呤包括 $9 \mathrm{H}$ 腺嘌呤和 $7 \mathrm{H}$ 腺嘌呤 (如 图 1 所示, “个”表示氢键供体位置, “ $\downarrow$ ”表示氢键受 体位置) ${ }^{10}$ 此外, 水、甲醇等作为溶剂会参与氢键网 络的构成, 从而形成了含水共晶体或含甲醇共晶 体, 如腺嘌呤与 2,6-二羟苯甲酸含水共晶体、 ${ }^{5}$ 腺嘌 呤与已二酸含甲醇共晶体 ${ }^{8}$ 及腺嘌呤与草酸含水共 晶体 ${ }^{9}$ 等. 溶剂参与氢键网络的构成会打断腺嘌呤与 共晶形成物(CCF)之间的直接氢键形成, 不利于进 一步研究腺嘌呤与 CCF 之间的相互作用.

有关研究表明, $9 \mathrm{H}$ 腺嘌呤在气相中或水溶液中

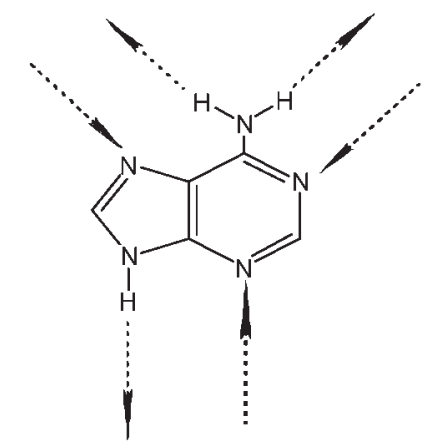

$9 \mathrm{H}$

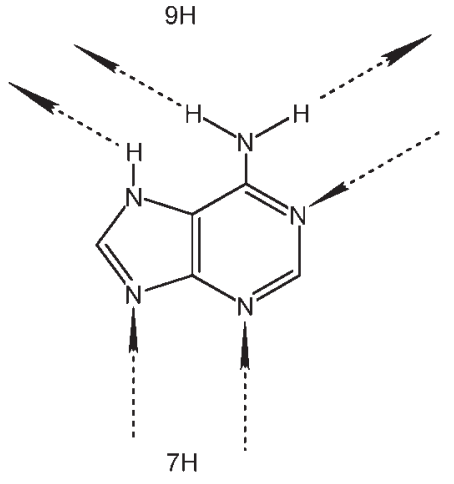

图 $19 \mathrm{H}$ 和 $7 \mathrm{H}$ 腺嘌呤受体与供体的可能位置

Fig1 Possible positions of donor and accepter for $9 \mathrm{H}$ and $7 \mathrm{H}$ adenine
是腺嘌呤同分异构体中最稳定的结构, ${ }^{10-12}$ 而 $9 \mathrm{H}$ 腺 嘌呤与 $7 \mathrm{H}$ 腺嘌呤之间的能量差在气相中为 $29.3-$ $33.5 \mathrm{~kJ} \cdot \mathrm{mol}^{-1}$, 而且在极性溶液中两者之间的能量 差值会减少. ${ }^{13,14}$ 针对上述问题, 本实验采用极性低 于水与甲醇的无水乙醇作为溶剂制备溶液共晶体, 降低溶剂引起腺嘌呤异构体之间转变的可能性, 同 时制备研磨共晶体, 与溶液共晶体对比, 观察溶剂 是否参与氢键形成. 选用富马酸作为 $\mathrm{CCF}$ 与腺嘌呤 形成共晶体, 是基于富马酸包含双羧基, 有利于与 腺嘌呤的氢键受体、供体形成双位氢键, 更易于氢 键网络的形成.

太赫兹波段位于微波和红外辐射之间, 其频段 为 $0.1-10 \mathrm{THz}$ (频率为 $1 \mathrm{THz}$ 的光子能量为 4.1 $\mathrm{meV}),{ }^{15}$ 与大部分有机分子及分子团的振动和转动 能级之间跃迁的能量大致相当. 相对于 $\mathrm{X}$ 射线衍射 技术能量高, 可能引起晶型转变的缺点, 太赫兹时域 光谱(THz-TDS)技术具有无损、快速检测的优点. 近 年来, 太赫兹时域光谱技术已被用于共晶检测中. ${ }^{16-18}$

本文制备了以乙醇为溶剂的腺嘌呤与富马酸 溶液共晶体和研磨共晶体, 采用太赫兹时域光谱技 术对腺嘌呤、富马酸与两者的溶液共晶体及研磨共 晶体进行表征. 通过谱图对比, 确认了共晶体的形 成, 并分析乙醇是否参与了共晶体的氢键形成. 运 用密度泛函理论对腺嘌呤与富马酸共晶体形成的 三种可能结构进行模拟, 结合理论模拟结果确认共 晶体结构, 并对共晶体的特征峰进行振动模式归属.

\section{2 实 验}

\section{1 样品制备}

腺嘌呤、富马酸均购于西格玛奥德里奇(上海) 贸易有限公司, 纯度均大于 $99 \%$, 使用前未进行进 一步的纯化.

\subsection{1 溶液共晶体的制备}

使用精度为 $0.0001 \mathrm{~g}$ 的电子天平(BSA-124S, 北 京赛多利斯科学仪器有限公司)分别称取摩尔比为 1 : 1 的腺嘌呤及富马酸, 将其溶于温热的无水乙醇中, 冷却至室温, 自然蒸发即可得到溶液共晶体样品.

\subsection{2 研磨共晶体的制备}

分别将两种药品在研钵中研磨成细小颗粒, 然 后使用电子天平称取摩尔比为 $1: 1$ 的腺嘌呤与富马 酸, 并利用漩浴混合器(QL-901, 海门市麒麟医用仪 器厂)震荡 $15 \mathrm{~min}$, 以确保混合充分, 然后将样品放 入行星式球磨机(QM-3SP04, 南京大学仪器厂)内, 
在频率 $25 \mathrm{~Hz}$ 下研磨 $120 \mathrm{~min}$ 获得研磨共晶体.

\section{2 样品表征}

THz-TDS 采用(美国 Zomega 公司)Z2 测量系 统. 激发光源为 Spectra Physics 公司的钛蓝宝石飞 秒锁模脉冲激光器, 激光重复频率 $80 \mathrm{MHz}$ 、脉宽 $100 \mathrm{fs}$ 、中心波长 $800 \mathrm{~nm}$. 使用 FW-4 型压片机(天津 天光光学仪器有限公司)将样品在 $4 \mathrm{MPa}$ 的压力下 压制成直径为 $13 \mathrm{~mm}$ 、厚度为 $1.5-1.7 \mathrm{~mm}$ 无裂缝且 两端面平行的样片. 样品测试在室温下进行, 且使 用氮气进行除湿, 使样品腔相对湿度保持在 $0 \%$.

\section{3 理论计算}

本文采用 Gaussian 03 软件 ${ }^{19}$ 分别对腺嘌呤、富 马酸及两者共晶体进行分子模拟, 选取密度泛函理 论 B3LYP 方法, ${ }^{20,21}$ 结合基组 6-31G $(d, p)$ 进行理论计 算. 模拟计算中, 首先进行结构优化, 再进行频率计 算. 所有样品的计算结果没有出现虚频, 说明所有 优化都找到了分子最小能量结构. 考虑到模拟中没 有完全考虑电子相关作用和其对非简谐性效应的 忽略, 以及基组选择等因素, 采用相应矫正因子 $0.96 .{ }^{22}$ 实验结果显示溶液与研磨共晶体的物相结构 相同, 由此判断乙醇并未参与氢键形成. 根据上文 分析及 Thompson 等 ${ }^{4}$ 实验结果可确定参与共晶体 氢键形成的是 $9 H$ 腺嘌呤. 同时依据氢键合成元(羧 酸-羧酸、羧酸-吡啶、羧酸-酰胺、醇-吡啶、醇-胺 $)^{23}$ 和 $9 \mathrm{H}$ 腺嘌呤的氢键供体和受体的位置, 理论认为 $9 \mathrm{H}$ 腺 嘌呤与富马酸共晶体的单分子结构如图 2 所示, 图 $2 \mathrm{~A}$ 中 $9 \mathrm{H}$ 腺嘌呤六元环的 $\mathrm{N} 1$ 与富马酸羧基的 $-\mathrm{O} 1 \mathrm{H}$ 形成第一处氢键, 而这个羧基的 $\mathrm{C} 1=\mathrm{O} 2$ 与 $\mathrm{N} 10-\mathrm{H}$ 形成第二处氢键, 由此形成腺嘌呤与富马酸共晶体 的双位氢键; 图 $2 \mathrm{~B}$ 中富马酸的 $\mathrm{C} 1=\mathrm{O} 2$ 与 $\mathrm{N} 10-\mathrm{H}$ 形成第一处氢键, 且此羧基的 $\mathrm{O} 1 \mathrm{H}$ 与腺嘌呤五元环 上的 $\mathrm{N} 7$ 形成第二处氢键; 图 $2 \mathrm{C}$ 中 $\mathrm{C} 1=\mathrm{O} 2$ 与腺嘌 呤五元环上的 $\mathrm{N} 9-\mathrm{H}$ 形成第一处氢键, 而 $\mathrm{O} 1 \mathrm{H}$ 与腺 嘌呤六元环上的 N3 则形成第二处氢键.

\section{4 结果与分析}

腺嘌呤与富马酸共晶体的太赫兹谱如图 3 所 示. 图 $3 \mathrm{~A}$ 为腺嘌呤与富马酸溶液共晶体和其研磨共 晶体的光谱结果比较, 从图中可看出腺嘌呤与富马 酸溶液共晶体和其研磨共晶体在太赫兹谱图中特征 峰出现的位置相同, 表明两者物相结构一致, 且说明 了乙醇并未参与氢键的形成. 图 3B 为腺嘌呤与富马
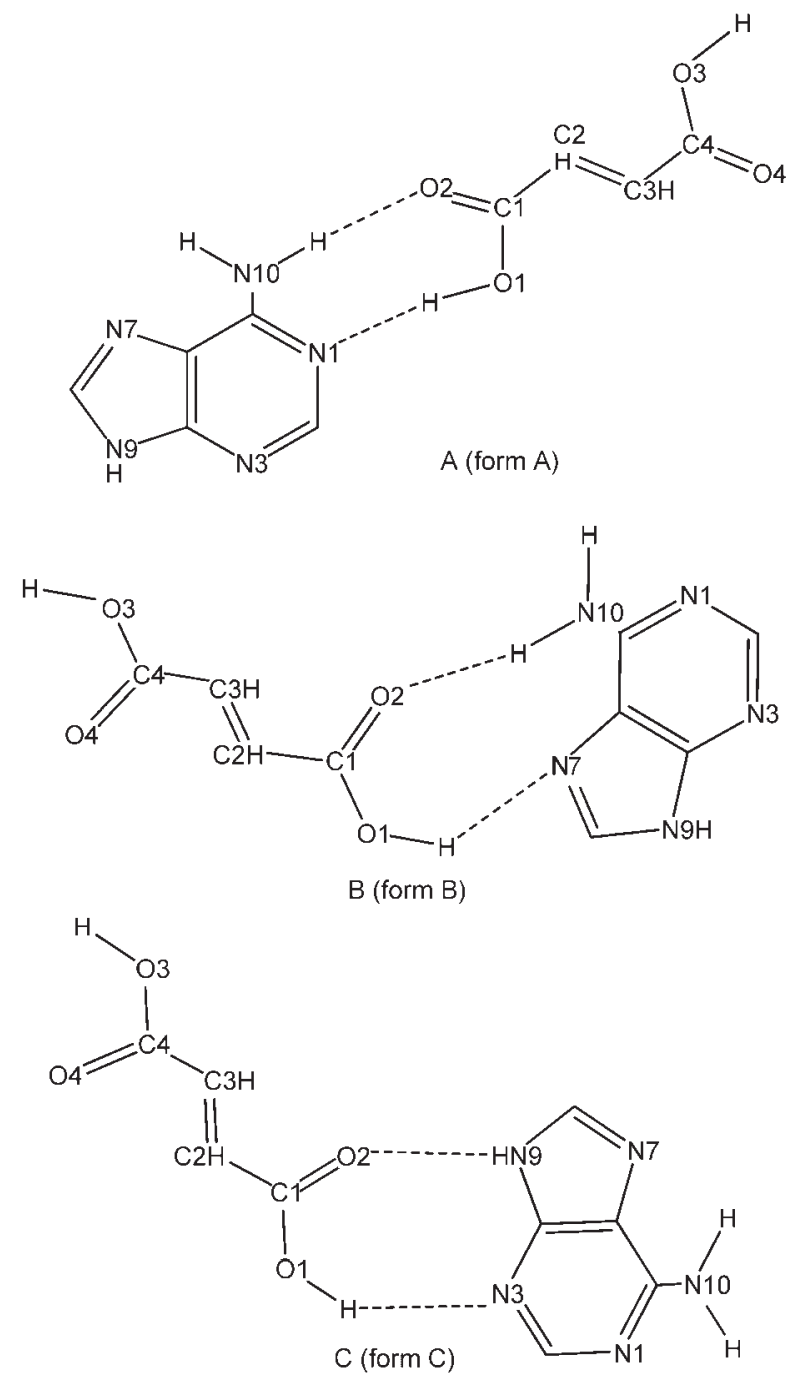

图 29 腺嘌呤与富马酸三种共晶体理论晶型

Fig 2 Three theoretical structures of cocrystal between 9H adenie and fumaric acid

酸共晶体及其原料的太赫兹吸收谱对比. 由图 3B 可 知, 相对于原料, 共晶体的太赫兹谱图在 0.92、1.24、 $1.52 \mathrm{THz}$ 处都有明显的吸收峰, 与原料不同.

理论模拟与实验谱图对比如图 4 所示, 共晶体 理论晶型 $\mathrm{A}$ 相对于另外两种共晶体理论晶型(B 和 C)的光谱模拟结果与实验所得共晶体太赫兹吸收 谱图更相符. 共晶体理论晶型 $\mathrm{A}$ 的特征吸收峰位于 $0.89 、 1.16 、 1.41 \mathrm{THz}$ 处, 对应共晶的实验吸收峰位于 $0.92 、 1.24 、 1.52 \mathrm{THz}$ 处, 因此认为腺嘌呤与富马酸共 晶体结构为理论模拟中的晶型 $\mathrm{A}$ 构型, 其结构如图 5 所示. 这一结果与 Thompson 等 ${ }^{4}$ 使用 $\mathrm{X}$ 射线衍射 检测所得结果一致. 氢键的形成对于分子内键长、 键角的影响如表 $1 、 2$ 所示(实验数据见文献 ${ }^{24,25}$ ), 氢

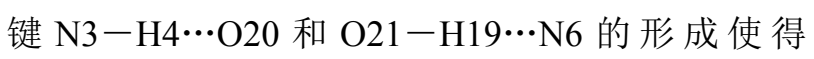
$\mathrm{N} 3-\mathrm{H} 4$ 的键长由 $0.1007 \mathrm{~nm}$ 伸长至 $0.1023 \mathrm{~nm}$; 


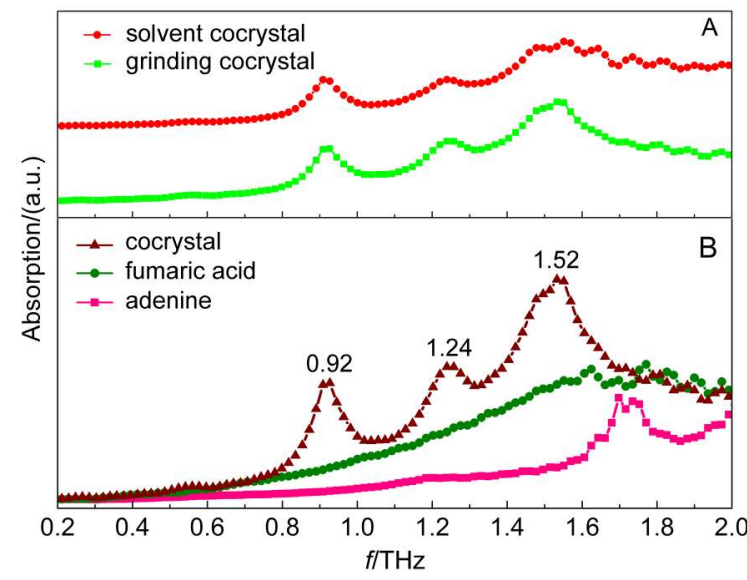

图 3 腺嘌呤、富马酸及两者共晶体的太赫兹谱图

Fig 3 THz spectra of adenine, fumaric acid, and cocrystals (A) solvent and grinding cocrystal; (B) adenine, fumaric acid, and cocrystal

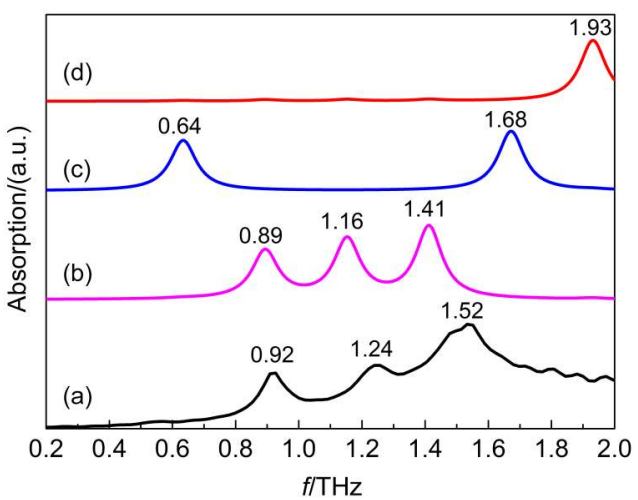

图 4 腺嘌呤与富马酸共晶体理论结构 $\mathrm{A}$ 型, $\mathrm{B}$ 型, $\mathrm{C}$ 型 太赫兹吸收光谱与实验结果对比

Fig4 Comparison of experimental and form A, form B, form $\mathrm{C}$ theoretical $\mathrm{THz}$ absorption spectra of cocrystal between adenie and fumaric acid

(a) experimental result; (b) theoretical form A; (c) theoretical form B; (d) theoretical form $\mathrm{C}$

C9… N6 的键长由 $0.1345 \mathrm{~nm}$ 伸长至 $0.1358 \mathrm{~nm}$; $\mathrm{O} 21-\mathrm{H} 19$ 的键长由 $0.0972 \mathrm{~nm}$ 伸长至 $0.1028 \mathrm{~nm}$; 原子之间的键长变长使得原有振动模式的频率降
表 1 腺嘌呤与富马酸中氢键的键长变化

Table 1 Change of hydrogen bond length between adenine and fumaric acid

\begin{tabular}{|c|c|c|c|c|c|}
\hline \multirow{3}{*}{$\begin{array}{c}\text { Chemical } \\
\text { bond }\end{array}$} & \multicolumn{5}{|c|}{ Bond length /nm } \\
\hline & \multicolumn{2}{|c|}{ Experimental data } & \multicolumn{3}{|c|}{ Calculation values } \\
\hline & adenine $^{24}$ & fumaric acid ${ }^{25}$ & adenine & fumaric acid & cocrystal \\
\hline $\mathrm{N} 3-\mathrm{H} 4$ & 0.0877 & - & 0.1007 & - & 0.1023 \\
\hline $\mathrm{C} 9 \ldots \mathrm{N} 6$ & 0.1380 & - & 0.1345 & - & 0.1358 \\
\hline $\mathrm{O} 21-\mathrm{H} 19$ & - & 0.1080 & - & 0.0972 & 0.1028 \\
\hline $\mathrm{C} 17-\mathrm{O} 21$ & - & 0.1287 & - & 0.1355 & 0.1319 \\
\hline
\end{tabular}

低, 即红移现象, 这也是共晶体的太赫兹谱图(图 3) 中 0.92、1.24 THz 处两个新峰产生的原因. 而 $\mathrm{C} 17-$ $\mathrm{O} 21$ 的键长则由 $0.1355 \mathrm{~nm}$ 缩短至 $0.1319 \mathrm{~nm}$. 此外, 参与氢键形成的化学键角都有所增大, 从而也使得 共晶体的振动光谱图区别于原料样品. 模拟和实验 结果之间键长的差别和特征吸收峰存在少许偏移, 都是因为实验是在常温下进行的, 而理论模拟是在 绝对零度下进行的, 没有考虑热效应; 且实验结果 是整个晶格的振动, 而计算模拟仅仅针对单个共晶 体分子.

分子在太赫兹低频波段的振动主要源于多个 原子参与的变形振动、扭动振动和弯曲振动 ${ }^{26}$ 或氢 键作用引起的相对振动, 且不同峰位的振动模式不 同. 通过 GaussianView 的动态观察, 可对腺嘌呤与 富马酸共晶体的振动模式进行大致的归属, 结果见 表 3. 腺嘌呤与富马酸共晶体在 $0.92 \mathrm{THz}$ 处的吸收 峰是由富马酸的碳链 $\mathrm{C} 23-\mathrm{C} 22=\mathrm{C} 16-\mathrm{C} 17$ 集体面 外摇摆振动和腺嘌呤的氨基 $\mathrm{H} 4-\mathrm{N} 3-\mathrm{H} 5$ 面外摇摆 振动共同引起的, 如图 6(a)所示; 在 $1.24 \mathrm{THz}$ 处的吸 收峰是由 $\mathrm{O} 25=\mathrm{C} 23-\mathrm{O} 26$ 面内弯曲振带动共晶体 的氢键 $\mathrm{O} 21-\mathrm{H} 19 \cdots \mathrm{N} 6$ 振动, 并以 $\mathrm{O} 21-\mathrm{H} 19 \cdots \mathrm{N} 6$ 为连接轴引起腺嘌呤分子面内弯曲振动所共同作 用引起的, 如图 6(b)所示; 在 $1.52 \mathrm{THz}$ 处的吸收峰则 是由富马酸的两个羧基 $\mathrm{O} 25=\mathrm{C} 23-\mathrm{O} 26, \mathrm{O} 20=$

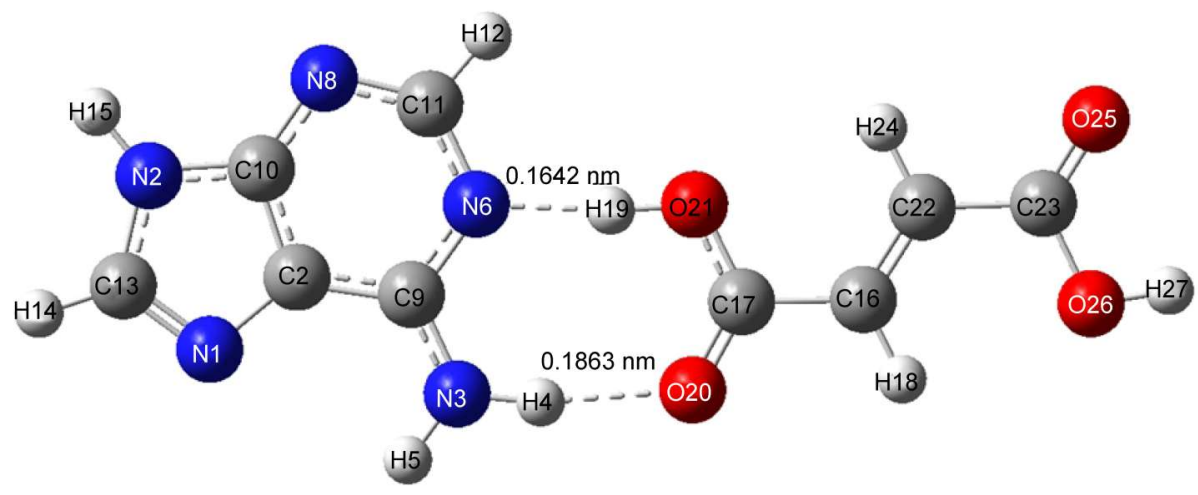

图 5 腺嘌呤与富马酸理论共晶体晶型 $\mathbf{A}$ 模拟优化结构

Fig5 Optimized structure of theoretical cocrystal form $A$ between adenie and fumarinc acid 
表 2 腺嘌呤与富马酸中氢键的键角变化

Table 2 Change of bond angle length between adenine and fumaric acid

\begin{tabular}{|c|c|c|c|c|c|}
\hline & \multicolumn{5}{|c|}{ Bond angle $/\left({ }^{\circ}\right)$} \\
\hline & \multicolumn{2}{|c|}{ Experiment data } & \multicolumn{3}{|c|}{ Calculation values } \\
\hline & $\operatorname{adenine}^{24}$ & fumaric $\operatorname{acid}^{25}$ & adenine & fumaric acid & cocrystal \\
\hline$\angle \mathrm{H} 5-\mathrm{N} 3-\mathrm{H} 4$ & 123.6 & - & 120.7 & - & 121.2 \\
\hline$\angle \mathrm{C} 9-\mathrm{N} 6-\mathrm{C} 11$ & 119.2 & - & 118.3 & - & 119.5 \\
\hline$\angle \mathrm{H} 19-\mathrm{O} 21-\mathrm{C} 17$ & - & 112.2 & - & 106.1 & 117.8 \\
\hline$\angle \mathrm{O} 21-\mathrm{C} 17-\mathrm{O} 20$ & - & 124.4 & - & 123.0 & 125.2 \\
\hline
\end{tabular}

表 3 腺嘌呤与富马酸共晶体的振动模式

Table3 Vibrational modes of cocrystal between adenine and fumaric acid

\begin{tabular}{ccl}
\hline No. $^{*}$ & $f / \mathrm{THz}$ & \multicolumn{1}{c}{ Vibrational mode } \\
\hline $\mathrm{a}$ & 0.92 & $\mathrm{C} 23-\mathrm{C} 22=\mathrm{C} 16-\mathrm{C} 17$ torsion; $\mathrm{H} 4-\mathrm{N} 3-\mathrm{H} 5$ out of plane vibration \\
$\mathrm{b}$ & 1.24 & $\mathrm{O} 25=\mathrm{C} 23-\mathrm{O} 26$ in-plane bending vibration; $\mathrm{O} 21-\mathrm{H} 19 \cdots \mathrm{N} 6$ in-plane bending vibration \\
$\mathrm{c}$ & 1.52 & $\mathrm{O} 25=\mathrm{C} 23-\mathrm{O} 26, \mathrm{O} 20=17 \mathrm{C}-\mathrm{O} 21$ torsion; $\mathrm{H} 4-\mathrm{N} 3-\mathrm{H} 5$ out of plane vibration; O21 $-\mathrm{H} 19 \cdots \mathrm{N} 6$ torsion \\
\hline
\end{tabular}

*Nos. a-c correspond to the vibrational modes shown in Fig.6.

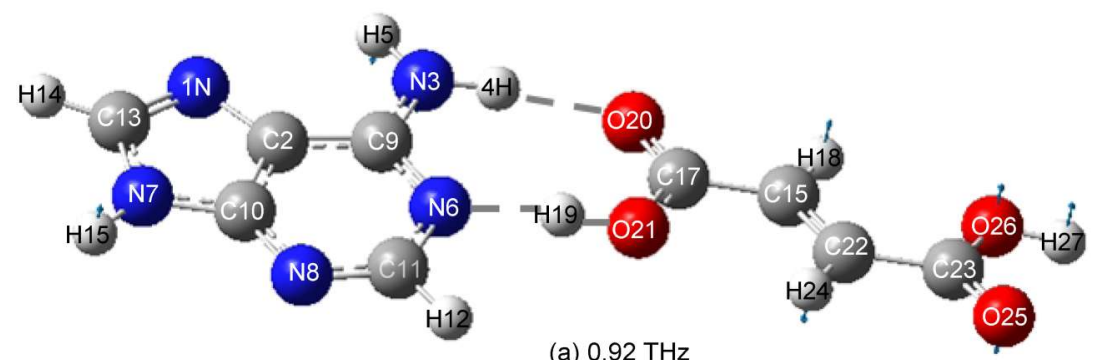

(a) $0.92 \mathrm{THz}$

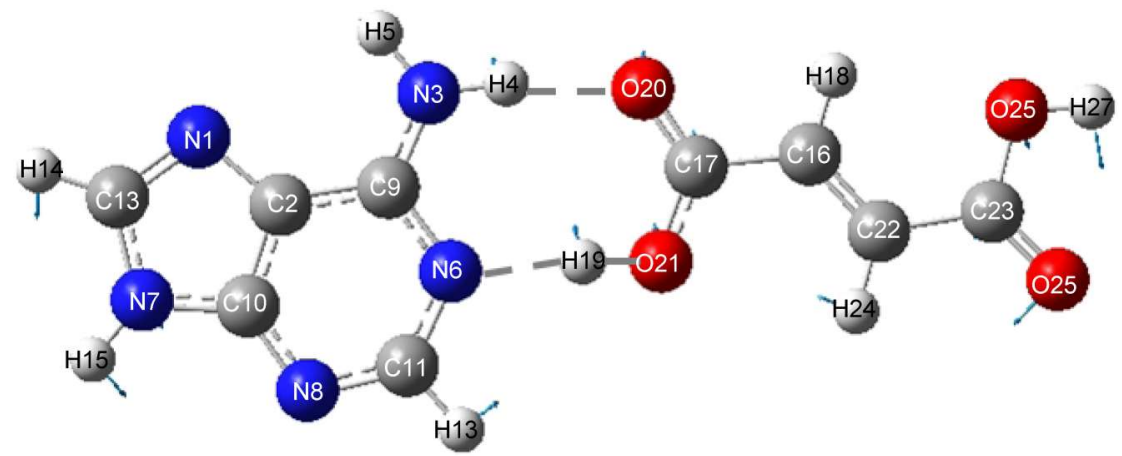

(b) $1.24 \mathrm{THz}$

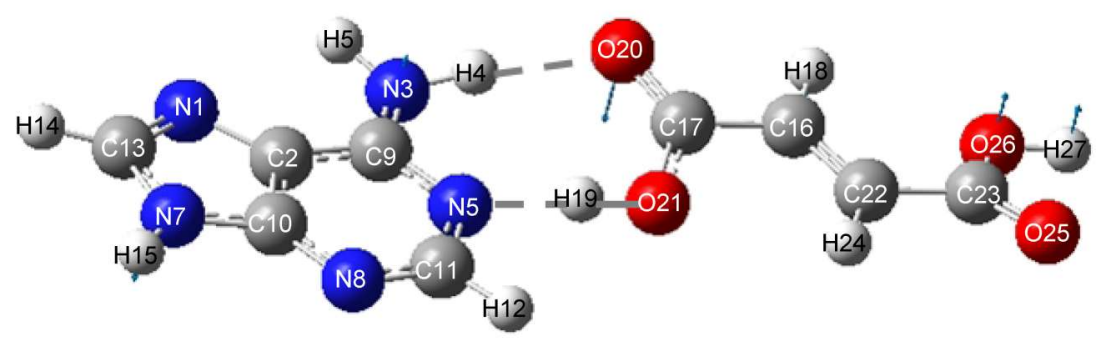

(c) $1.52 \mathrm{THz}$

图 6 腺嘌呤与富马酸共晶体位于 $0.92,1.24,1.52 \mathrm{THz}$ 处的振动模式

Fig 6 Vibrational modes of cocrystal between adenie and fumaric acid at $0.92,1.24,1.52 \mathrm{THz}$ 
$17 \mathrm{C}-\mathrm{O} 21$ 扭曲振动, 并以 $\mathrm{O} 21-\mathrm{H} 19 \cdots \mathrm{N} 6$ 为轴使得 腺嘌呤分子产生面外摇摆振动所引起的, 而腺嘌呤 的 H4-N3-H5 面外摇摆振动加深了这一作用, 如 图 6(c)所示. 此外, 根据计算结果腺嘌呤与富马酸共 晶体在 $3.05 \mathrm{THz}$ 处有一个以氢键 $\mathrm{O} 21-\mathrm{H} 19 \cdots \mathrm{N} 6$ 为 轴的两个分子共同作用的剪式振动所产生的吸收 峰, 但在实验中由于仪器光谱有效频段范围的限制 未能在实验谱图中体现.

根据实验及计算结果可知, 氢键的形成使得腺 嘌呤分子与富马酸分子内部结构有一些变化, 且在 各自的振动模式下受到对方的影响, 这也使得腺嘌 呤与富马酸共晶体在太赫兹波段呈现出和原料完 全不同的特征吸收谱.

\section{5 结 论}

使用 THz-TDS 技术在室温下对腺嘌呤、富马酸 及两者的溶液和研磨共晶体进行表征和分析, 发现 腺嘌呤与富马酸的溶液共晶体与研磨共晶体在 $0.92 、 1.24$ 及 $1.52 \mathrm{THz}$ 处都有明显且区别于原料样 品的吸收峰; 由于两种方法得到的物相结构一致, 从而说明乙醇作为弱极性溶剂并未参与氢键的形 成. 采用密度泛函理论对腺嘌呤与富马酸由氢键作 用而形成共晶体的三种可能结构进行结构优化与 光谱模拟, 并将计算结果与实验结果进行对比, 发 现其中共晶体理论晶型 $\mathrm{A}$ 的模拟结果与实验太赫 兹谱图结果吻合度很高, 据此推断共晶体的氢键形 成位置为腺嘌呤的氨基与富马酸其中一个羧酸的 碳氧双键形成一处氢键, 而此羧酸的羟基与腺嘌呤 六元环上的邻位氮原子形成第二处氢键. 该结论与 $\mathrm{X}$ 射线衍射结果一致. 为太赫兹光谱技术检测和分 析共晶体结构及氢键作用提供了实验与理论参考.

\section{References}

(1) Verma, R. P.; Hansch, C. J. Pharm. Sci. 2008, 97 (1), 88.

(2) Ohara, K.; Smeitana, M.; Restouin, A.; Mollard, S.; Borg, J. P.; Collette, Y.; Vasseur, J. J. J. Med. Chem. 2007, 50 (26), 6465. doi: 10.1021/jm701207m

(3) Song, Y. L.; Li, Y. L.; Wu, Z. Y. J. Inorg. Biochem. 2008, 102 (9), 1691. doi: 10.1016/j.jinorgbio.2008.04.005

(4) Thompson, L. J.; Elias, N.; Male, L.; Tremayne, M. Cryst. Growth Des. 2013, 13 (4), 1464. doi: 10.1021/cg301561j
(5) Byres, M.; Cox, P. J.; Kay, G.; Nixon, E. CrystEngComm 2009, 11 (1), 135. doi: 10.1039/b811243f

(6) Perumalla, S. R.; Suresh, E.; Pedireddi, V. R. Angew. Chem. Int. Edit. 2005, 44 (47), 7752.

(7) Du, L. C.; Zeng, W. L.; Liu, X. Y.; Jian, F. F. Acta Crystallogr., Sect. E: Struct. Rep. Online 2009, 65 (Suppl. 8), o1791.

(8) Sridhar, B.; Ravikumar, K.; Varghese, B. Acta Crystallogr., Sect. C: Cryst. Struct. Commun. 2009, 65 (Suppl. 5), o202.

(9) McHugh, C.; Erxleben, A. Cryst. Growth Des. 2011, 11 (11), 5096. doi: 10.1021/cg201007m

(10) Hanus, M.; Kabelacek, M.; Rejnek, J.; Ryjacek, F.; Hobza, P. J. Phys. Chem. B 2004, 108 (6), 2087. doi: 10.1021/jp036090m

(11) Lin, J.; Yu, C.; Peng, S.; Akiyama, I.; Li, K.; Lee, L. K.; LeBreton, P. R. J. Am. Chem. Soc. 1980, 102 (14), 4627. doi: 10.1021/ja00534a010

(12) Dreyfus, M.; Dodin, G.; Bensaude, O.; Dubois, J. E. J. Am. Chem. Soc. 1975, 97 (9), 2369. doi: 10.1021/ja00842a011

(13) Gu, J. D.; Leszczynski, J. J. Phys. Chem. A 1999, 103 (15), 2744. doi: 10.1021/jp982713y

(14) Huang, Y. Q.; Kenttamaa, H. J. Phys. Chem. A 2004, 108 (20), 4485. doi: $10.1021 / \mathrm{jp} 0312767$

(15) Xu, J. Z.; Zhang, X. C. Technology and Applications of Terahertz Wave; Peking University Press: Beijing, 2007; pp 15. [许景周, 张希成. 太赫兹波科学技术与应用. 北京: 北京大 学出版社, 2007: 1-5]

(16) Du, Y.; Xia, Y.; Zhang, H. L.; Hong, Z. Spectrochim. Acta A 2013, 111, 192. doi: 10.1016/j.saa.2013.03.081

(17) Nguyen, K. L.; Friscic, T.; Day, G. M.; Gladden, L. F.; Jones, W. Nat. Mater. 2009, 6 (3), 206

(18) Yang, C. X.; Wang, J.; Zhang, Z. W. Spectrosc. Spec. Anal. 2011, 31 (9), 2476. [杨彩霞, 王 静, 张振伟. 光谱学与光谱 分析, 2011, 31 (9), 2476.]

(19) Frisch, M. J.; Trucks, G. W.; Schlegel, H. B.; et al. Gaussian 03, Revision E.01; Gaussian, Inc.: Wallingford, CT, 2004.

(20) Axel, D. B. J. Chem. Phys. 1993, 98, 5648.

(21) Scoot, P.; Radom, L. J. Chem. Phys. 1996, 100, 16502.

(22) Zhao, R. J.; He, J. L.; Li, J.; Guo, C. S.; Du, Y.; Hong, Z. Acta Phys. -Chim. Sin. 2011, 27 (12), 2743. [ 赵容娇, 何金龙, 李 璟, 郭昌盛, 杜 勇, 洪 治. 物理化学学报, 2011, 27 (12), 2743.] doi: 10.3866/PKU.WHXB20112743

(23) Gao, Y.; Zhu, H.; Zhang, J. J. Prog. Chem. 2010, 22 (5), 829. [高 缘, 祖 卉, 张建军. 化学进展, 2010, 22 (5), 829.]

(24) Brown, C. J. Acta Crystallogr. 1966, $21,1$.

(25) Yu, B.; Zeng, F.; Yang, Y.; Xing, Q.; Chechin, A.; Xin, X.; Zeylikovich, I.; Alfano, R. R. Biophys. J. 2004, 86, 1649.

(26) Perumalla, S. R.; Suresh, E ; Pedireddi, V. R. Angew. Chem. Int. Edit. 2005, 44, 7752. 